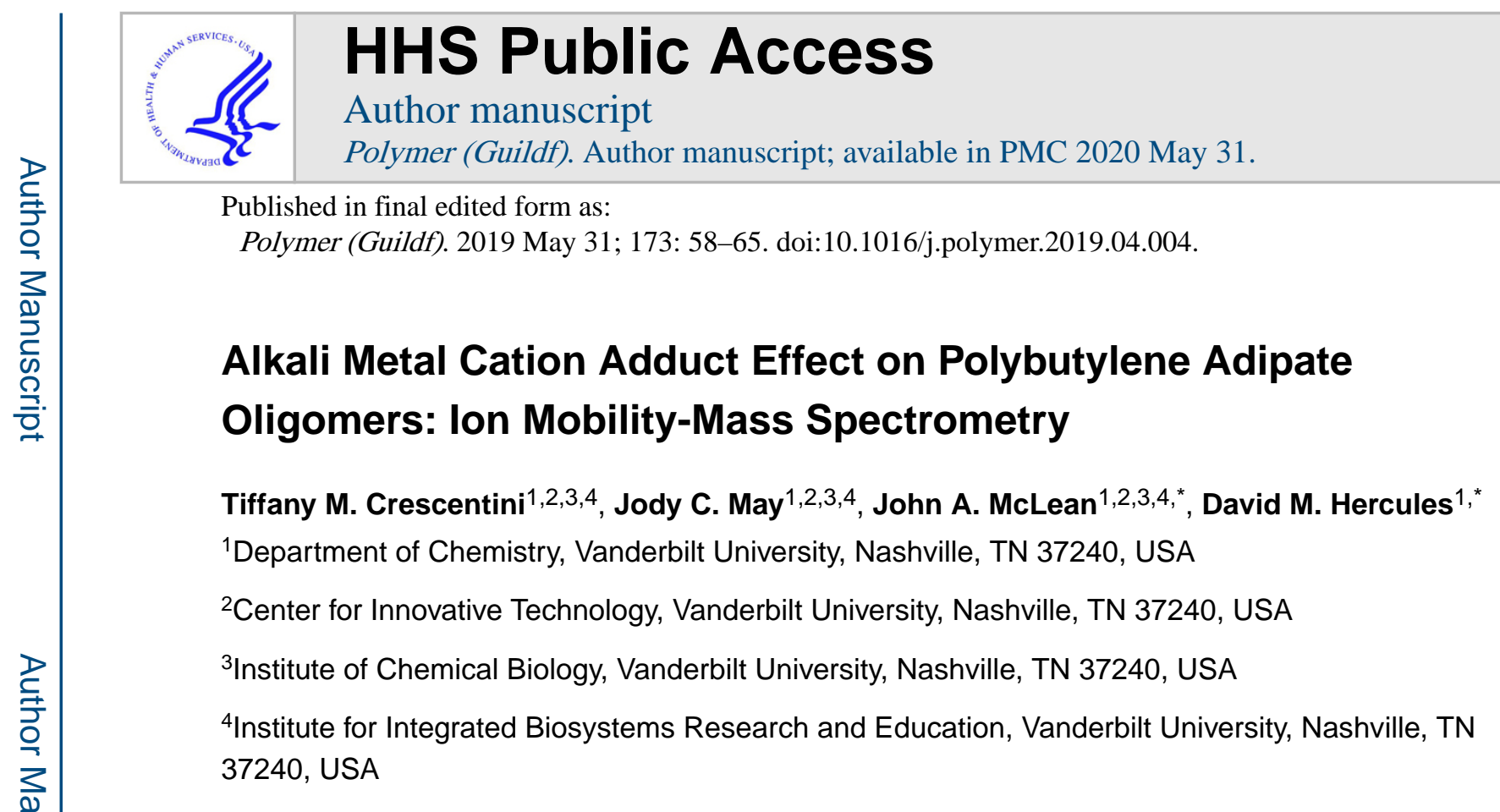

\title{
Abstract
}

Polyurethane (PU) di-block copolymers are one of the most versatile polymeric materials, comprised of hard and soft segments that contribute to PU's broad range of applications.

Polybutylene adipate (PBA) is a commonly used soft segment in PU systems. Characterizing the structure of PBA polymers is essential to understanding complex heterogeneity within a PU sample. In this study, ion mobility-mass spectrometry (IM-MS) and tandem mass spectrometry (MS/MS) are used to structurally characterize a PBA standard $\left(\mathrm{M}_{\mathrm{n}}=2250\right)$ adducted with a combination of monovalent alkali cations ( $\mathrm{Li}, \mathrm{Na}, \mathrm{K}, \mathrm{Rb}$, and $\mathrm{Cs}$ ). IM-MS profiles show unique trends associated with each cation-adducted PBA sample. Charge state trends: $+1,+2$, and +3 were extracted for cation-adducted PBA oligomers, and investigated to study gas-phase transitional folding. To quantitatively assess the gas-phase structural similarities and differences, a statistical test (ANOVA) was used to compare PBA oligomer-cation collisional cross sections (CCS). Fragmentation studies (MS/MS) identified the unique behavior of Li and $\mathrm{Na}$ for promoting 1,5 $\mathrm{H}$ shift and 1,3 H-shift fragmentation, whereas the PBA precursor preferentially loses the larger $\mathrm{K}$, $\mathrm{Rb}$, and $\mathrm{Cs}$ cations as the ion activation energy is increased. The combination of adducted alkali cations, IM-MS, and MS/MS allow for unique structural characterization of this important PBA system.

\section{Graphical Abstarct}

\footnotetext{
“Correspondence: john.a.mclean@ vanderbilt.edu; (J.A.M) and david.m.hercules@ vanderbilt.edu; (D.M.H).

Publisher's Disclaimer: This is a PDF file of an unedited manuscript that has been accepted for publication. As a service to our customers we are providing this early version of the manuscript. The manuscript will undergo copyediting, typesetting, and review of the resulting proof before it is published in its final citable form. Please note that during the production process errors may be discovered which could affect the content, and all legal disclaimers that apply to the journal pertain.
} 


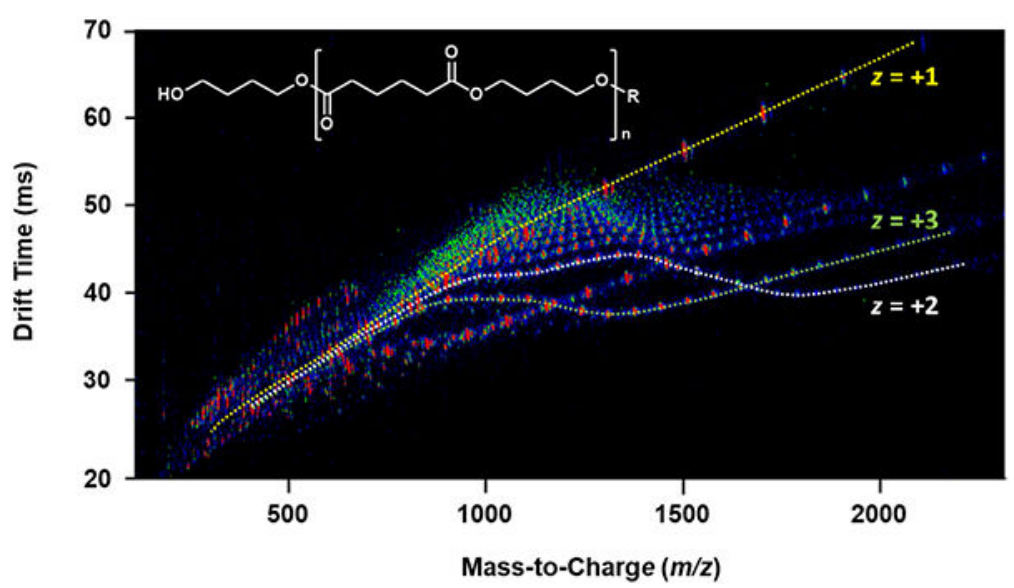

\section{Keywords}

Polybutylene adipate; alkali cations; degree of polymerization; ion mobility-mass spectrometry

\section{Introduction}

Polyurethanes (PUs) are one of the most versatile synthetic polymers and are well known for their applications in the automotive, construction, and medical industries. PUs are diblock copolymers composed of hard (urethane) and soft (aliphatic) segments which contribute to their diverse applications [1]. Hard segments such as methylene bisphenol diisocyanate (MDI) are known for their ridged urethane backbone when reacted with soft segment diols such as polyesters. When synthesizing PU polymers, polydispersity inevitably adds a level of complexity to a polymer sample [2].

Mass spectrometry (MS) has become one of the most useful analytical techniques for accurate mass measurement and detailed end-group analysis of synthetic polymers [3]. Coupling ion mobility (IM) separation to MS (IM-MS) can provide valuable information regarding the three-dimensional structure of a polymer's architectural motif. IM-MS is capable of differentiating isomeric species, such as linear and cyclic polymers [4,5], and can provide detailed characterization regarding ion size and mass. A molecule's size and shape are described quantitatively by the gas-phase collision cross section (CCS) derived from IM measurements [6,7]. Additionally, tandem mass spectrometry (MS/MS) promotes the structural characterization of a polymer's sequence. Coupling IM-MS to MS/MS provides multidimensional information that is useful for characterizing a polymer's molecular sequence and configured arrangement [8-11].

Another useful strategy which can be combined with IM-MS characterization is cation coordination. IM-MS cation coordination has been successfully utilized for biopolymers to analyze DNA base pair arrangements [12-14], carbohydrates [15,16] and peptide sequences [17]. Cation coordination for biopolymers has been extensively explored; however the application of cation coordination studies towards synthetic polymers using IM-MS has become a growing field in the last 25 years. Prior studies have noted that flexible poly(ethylene glycol) (PEG) oligomers encapsulate $\mathrm{Cs}^{+}$, coordinating up to 11 oxygen 
centers with the large $\mathrm{Cs}^{+}$cation to form a near spherical shape $[18,19]$. Other PEG studies have focused on low charge state polymer ions in order to characterize globular gas-phase geometries using both experimental and computational methods [20]. Other polymer systems such as poly(methyl methacrylate) were found to coordinate with various metal ions via the oxygen atoms along the polymer backbone [21], whereas polystyrene was discovered to coordinate with cations via $\pi$ stacking at its benzene groups, sandwiching the metal ions between the rings [22]. Several alkali ions were used to characterize poly(ethylene terephthalate) and the identity of the cation corresponded to the oligomer length [23]. Other polymer studies have investigated the addition of metal ions and their effect on polymer topology [24-27] and the stepwise folding process [28]. More recent studies investigating polymer-cation coordination have progressed towards identification of unique threedimensional structures; by studying these polymer-cation systems, the transition from an unfolded linear species to a folded globular conformation can be investigated [29]. For a given charge state, oligomer chain lengths are observed to fold around the cation in a welldefined manner. As the degree of polymerization (DP) increases for a given polymer, the structural motif was observed to transition towards a globular arrangement [30]. Uniquely, charge-induced unfolding of polymers has also been investigated [31].

In the present study, a telechelic PBA standard $\left(M_{n}=2250\right)$, Figure 1, was investigated by probing the effects of various Group I monovalent cations ( $\mathrm{Li}, \mathrm{Na}, \mathrm{K}, \mathrm{Rb}$, and $\mathrm{Cs}$ ). IM-MS studies identified multiple charge state $(z)$ trends for each cationized PBA oligomer. Unique transitional folding was noted for all charge state trends observed, including: $+1,+2$, and +3 . Empirical CCS values as obtained from uniform electric field measurements are reported for each alkali metal-adducted PBA oligomer. An analysis of variance (ANOVA) on the measured CCS values was used to determine gas-phase structural differences (different molecular volume) and structural similarities (same molecular volume) observed between each PBA-cation. IM-MS/MS was also utilized to study the unique fragmentation patterns associated with cation-oligomer species ( $\mathrm{DP}=1$ and $\mathrm{DP}=5$ ). Here the mobility-resolved ion fragmentation results revealed the unique behavior of the $\mathrm{Li}^{+}$and $\mathrm{Na}^{+}$cation to undergo $1,5 \mathrm{H}$-shift and 1,3 $\mathrm{H}$-shift fragmentation, compared to the larger cations $\mathrm{K}^{+}, \mathrm{Rb}^{+}$, and $\mathrm{Cs}^{+}$ which preferentially are lost upon increased activation energy. The combination of monovalent alkali metal cations, IM-MS, IM-MS/MS, and statistical strategies aided in the characterization of PBA's gas-phase structural behavior.

\section{Materials and Methods}

\subsection{Materials}

Optima LC-MS grade acetonitrile and water were purchased from Fisher Scientific (Waltham, MA). The PBA sample was provided by Bayer Material Science, with the number-average molecular weight $\left(M_{n}\right)$ determined by end-group analysis $\left(M_{n}=2250\right)$ [32]. MALDI analysis of this PBA sample determined $M_{n}=2155$ [33]. Lithium chloride, sodium chloride, potassium chloride, rubidium chloride, and cesium chloride were purchased from Sigma-Aldrich (St. Louis, MO). The PBA sample was prepared at an initial concentration of $1 \mathrm{mg} / \mathrm{mL}$ and subsequently diluted to a final concentration of $10 \mu \mathrm{g} / \mathrm{mL}$ in acetonitrile.

Group I monovalent cations were prepared at an initial concentration of $1 \mathrm{mg} / \mathrm{mL}$ in water. 
Then, metal cation solutions were diluted to $5 \mathrm{mM}$ in $10 \mu \mathrm{g} / \mathrm{mL}$ of the PBA sample in acetonitrile.

\subsection{Instrumentation}

All experiments were conducted on a commercially available drift tube IM-MS (6560-IMQTOF, Agilent Technologies, Santa Clara, CA, USA) as described previously [34]. The IM stage of this instrument consists of a uniform field drift tube $78.1 \mathrm{~cm}$ in length, filled with nitrogen and operated with a static drift gas pressure of ca. 4 Torr and $\sim 30{ }^{\circ} \mathrm{C}$. All samples were directly infused via a syringe pump (Cole-Palmer, Vernon Hills, IL) at a $10 \mu \mathrm{L} / \mathrm{min}$ flow rate into a thermally assisted ESI source (Agilent Jet Stream). The instrument was operated in the positive ion mode with $3.8 \mathrm{kV}$ applied to the ion transfer capillary and 1.8 $\mathrm{kV}$ applied to the ion focusing nozzle. The sample nebulizer is maintained at ground potential. Source temperatures were kept relatively low $\left(200{ }^{\circ} \mathrm{C}\right)$ to aid in ionization. Ion mobility parameters were adjusted to optimize the IM resolving power and utilize a gate width of $100 \mu$ s [35].

\subsection{CCS Measurements}

Collision cross sections $\left({ }^{\mathrm{DT}} \mathrm{CCS}_{\mathrm{N} 2}\right.$ ) were determined using a stepped field technique where the applied voltage across the drift tube was varied in 7 uniform increments from 1050 to $1650 \mathrm{~V}(7.0-19.8 \mathrm{~V} / \mathrm{cm}$ or $6-16 \mathrm{Td}$ at 4 Torr $)$ to determine the time ions reside outside the drift tube. The corrected drift times were then used to obtain CCS values based on the Mason-Schamp equation as described previously [34,36,37].

\subsection{Targeted MS/MS}

For targeted MS/MS, all ions were analyzed by IM and then $\mathrm{m} / \mathrm{z}$ species related to $\mathrm{DP}=1$ and $\mathrm{DP}=5[\mathrm{M}+\mathrm{Li}]^{+},[\mathrm{M}+\mathrm{Na}]^{+},[\mathrm{M}+\mathrm{K}]^{+},[\mathrm{M}+\mathrm{Rb}]^{+}$, and $[\mathrm{M}+\mathrm{Cs}]^{+}$oligomer adducts were mass isolated for CID (IM-MS/MS). For IM, the trap fill time was set to 60,000 $\mu$ s and 200 $\mu$ s for trap release time. For CID, a stepped voltage ramp was used with increments of $10 \mathrm{~V}$ between $0-100 \mathrm{~V}$ (laboratory frame). The IM-MS was tuned and mass calibrated using a commercially-available tuning mixture (ESI-L Tune Mix, Agilent).

\section{Results and Discussion}

\subsection{IM-MS Characterization}

In this study, we monitored the evolution of cationized PBA for changes in CCS as a function of DP for three different charge states: $+1,+2$, and +3 ions. These charge states were generated by coordination of monovalent alkali cations ( $\mathrm{Li}, \mathrm{Na}, \mathrm{K}, \mathrm{Rb}$, and $\mathrm{Cs}$ ) onto the polymer chain, and labeled accordingly in Figure 2. Due to polydispersity within the PBA sample, each charge state trend represents a range of PBA DP coordination with alkali metal cations. The full ESI-MS spectra of each PBA-cationized sample can be found in Supporting Information (Figures S1-S2). By using ESI to characterize PBA, we observe multiply charged species, which are characteristic of this technique. The majority of species contained in this PBA polymer sample were telechelic oligomers with - $\mathrm{OH}$ end groups. Within this PBA sample, only a few low MW cyclics were detected as seen in Supporting Information on Page S3 and in Figure S1. 
Figure 2 contains a plot of the CCS as a function of the PBA DP, increasing in chain length from left to right. For charge state +1 , we observe a mean constant increase in CCS as the PBA chain length increases for each cationized DP. To ensure that the IM profiles observed are unique to the PBA polymer sample alone and not to the instrument conditions, the IMMS ion funnel trap RF voltage was increased in $20 \mathrm{~V}$ increments from $80-200 \mathrm{~V}_{\mathrm{pp}}$ (Supporting Information Figure S3). Varying the trap RF voltage in this manner can probe for low energy structural transitions (i.e. polymer unfolding that would otherwise go unnoticed) [38]. There were no changes to the PBA charge state trends in the IM profile, respective to varying the trap RF voltage. As observed similarly by Haler et al. for poly(ethoxyphosphate) and poly(ethylene oxide) polymers [22], CCS evolution for higher charge state species (greater than +1 ) are known to have a defined structural transition at a specific DP. The structural transition can be characterized by a visual change in a CCS IM plot, and is attributed to a collapse of the polymer's gas-phase conformation to smaller CCS. The point of structural transition for this PBA system is observed at the beginning of the +2 and +3 charge state trend lines. This is where the polymer ion's chain length exceeds the coulombic forces induced by the multiple cations. The PBA species are then observed to behave predictably with a near linear increase in CCS respective to the increase in the polymers length. When the polymer cationizes with two metal adducts $(z=+2)$, a noticeable "tipping point" in CCS is observed between DP $=4-8$. Here the polymer species is observed to begin transitional folding around the two cations contained in the PBA ion structure. A similar region of structural folding is more dramatically observed for the higher +3 charge state trend line, where PBA coordinates with three cations. Here the tipping point in CCS is observed to reach its peak transition from DP $=7-14$. This transition is characterized by a decrease in CCS between DP $=15-19$ after which the CCS increases with polymer chain length in a linear trend for DP $\geq 20$. We hypothesize that this positive linear trend in CCS following the region of fold change indicates when the PBA chain wraps around the attached cations.

It is expected that longer PBA oligomer chains can accommodate more cations than shorter chains and this bears out in the observations made in this study. For instance, singly charged PBA oligomers are first observed for $\mathrm{DP}=1$, doubly charged species are observed at $\mathrm{DP}=$ 4 , and triply charged species begin to form at $\mathrm{DP}=7$. As the PBA chain length increases in size, the polymer's ability to coordinate with additional cation species increases. The full IM-MS profile of each PBA-cation sample can be found in Supporting Information (Figure S4).

\subsection{Structural Transition}

Figure 3 shows plots of the first derivative of $\operatorname{CCS}\left(\frac{\Delta C C S}{\Delta D P}\right)$ for each PBA-cation at charge states $+1,+2$, and +3 . Figure 3 a illustrates that the point of change is indicated by a valley, where the data indicates that a structural transition in a polymer's topology occurs respective to DP. The point of maximum slope change determined the region of structural change for each charge state trend. The point at which $\triangle \mathrm{CCS}$ converges to the smallest number represents the tipping point, that is, the point where the polymer chain wraps around the attached cations, previously referred to as "beads-on-a-string" [29]. In Figure $3 \mathrm{~b}-\mathrm{f}, \Delta \mathrm{CCS}$ was calculated for each PBA's cationized charge state trend and illustrated accordingly: +1 
(dotted-line), +2 (dashed-line), and +3 (solid-line). Blue dashed lines are drawn for visual alignment of the point of structural change represented by the negative apex for each charge state. Along the charge state +1 trend line, we observed minor transitional folding for PBA$\mathrm{Na}, \mathrm{PBA}-\mathrm{K}, \mathrm{PBA}-\mathrm{Rb}$, and PBA-Cs between DP $=2-3$, (Figure 3c-f). However, PBA-Li species were not observed to undergo any fold change in the charge state +1 trend for $\mathrm{DP}=$ $2-3$ (Figure $3 b$ ).

In Figure 3b-f, the valleys indicate that significant structural changes occur for charge states +2 and +3 . Table 1 summarizes the region of significant structural change along the +2 and +3 charge state trend lines as recorded for each cation: $\mathrm{Li}, \mathrm{Na}, \mathrm{K}, \mathrm{Rb}$, and $\mathrm{Cs}$. The +2 charge state structural transition region occurs between $\mathrm{DP}=6-10$ for all five cations. However, the +3 charge state structural transition region changes with respect to cation size. In Table 1 , the +3 charge state structural transitions for Li start at chain length $\mathrm{DP}=11$. As the cation size increases to $\mathrm{Na}$ and $\mathrm{K}$, the region of structural transition starts at DP $=12$. Similarly, the larger cations $\mathrm{Rb}$ and $\mathrm{Cs}$ undergo structural changes at a longer chain length of $\mathrm{DP}=13$. This pattern highlights the effect that cation size has on the polymer's gas-phase structure. Although we observed these cation species to undergo structural change at different DP values, all cations were found to end their transitions at DP $=20$. The structural transition region for Li spanned a range of 10 PBA DPs, Na and K spanned 9 PBA DPs, and Rb and Cs transitioned across only 8 PBA DP additions. In summary, the region of structural change is expected for multiply charged ions (charge state +2 and +3 ) due to the equilibrium between solvation of the charges and Coulomb repulsion, however in this study we observed slight structural change occurring for some of the cations within the charge state +1 trend line.

Table 1 also shows the tipping points for each PBA-cation at charge states +2 and +3 . Here, no large differences were observed in the structural transition between charge states +2 and +3 . The tipping point, or point of greatest change for charge state +2 was observed to be DP $=7$ for all five cations. The tipping point for charge state +3 occurred at DP $=17$ for all cation species. As stated above, we observed the PBA region of structural folding to be directly related to cation coordination and size. As a general observation, the smaller cations, such as $\mathrm{Li}, \mathrm{Na}$, and $\mathrm{K}$ were found to start transition folding at a shorter PBA chain length, compared to $\mathrm{Rb}$ and $\mathrm{Cs}$.

\subsection{Ion Mobility Plots}

IM distributions of PBA-cationized by Li (green), $\mathrm{Na}$ (blue), $\mathrm{K}$ (red), $\mathrm{Rb}$ (purple), and $\mathrm{Cs}$ (yellow) are shown in Figure 4 for $\mathrm{DP}=1,3$, and 5 at charge state +1 . The corresponding CCS means for each PBA species and their respective standard deviations are represented by error bars at the top of each mobility trace. The error bars on each mobility trace shows both the positive and negative standard deviation CCS values for each species. The vertical dashed lines are drawn for visual alignment within Figure $4 \mathrm{a}-\mathrm{c}$ and represent the maximum and minimum CCS values (means) observed for all five PBA-cation species. In Figure 4a, PBA-cation species $(\mathrm{DP}=1)$ have a CCS range that spans approximately $18 \AA^{2}$ : PBA-Li $=$ $163 \pm 0.4 \AA^{2}, \mathrm{PBA}-\mathrm{Na}=166 \pm 0.3 \AA^{2}, \mathrm{PBA}-\mathrm{K}=171 \pm 0.4 \AA^{2}, \mathrm{PBA}-\mathrm{Rb}=172 \pm 0.8 \AA^{2}$, and PBA-Cs $=174 \pm 0.5 \AA^{2}$. As cation size increases from Li to Cs, we observed a 
corresponding increase in CCS for PBA-cation species known as a periodic trend. In Figure $4 \mathrm{~b}$, for a PBA chain length of $\mathrm{DP}=3$, the maximum and minimum CCS values are observed to span $8 \AA^{2}$. It is interesting to note that for $\mathrm{DP}=3$, the CCS values are much closer to one another and do not follow a periodic trend. Here, PBA-Na has a smaller mean CCS than PBA-Li: PBA-Li $=249 \pm 0.3 \AA^{2}$, PBA-Na $=247 \pm 0.4 \AA^{2}$, PBA-K $=250 \pm 0.3 \AA^{2}$, PBA-Rb $=252 \pm 0.8 \AA^{2}$, and PBA-Cs $=255 \pm 0.5 \AA^{2}$. In Figure $4 \mathrm{c}$, for chain length of DP = 5, the observed CCS range maximum and minimum values converge toward the same CCS, a $2 \AA^{2}$ range. This is within the scale of the standard deviation of the measurements, and there are no periodic trends that can be derived from these measurements. The corresponding PBA CCS values are: PBA-Li $=321 \pm 0.5 \AA^{2}$, PBA-Na $=321 \pm 0.7 \AA^{2}$, PBA-K $=322 \pm 0.4 \AA^{2}$, $\mathrm{PBA}-\mathrm{Rb}=323 \pm 0.8 \AA^{2}$, and PBA-Cs $=322 \pm 0.5 \AA^{2}$. Taken collectively, these observations indicate that as the PBA DP increases from DP $=1$ to $\mathrm{DP}=5$, the PBA-cationized CCS values converge. This convergence in CCS is attributed to the polymer's structural transition. As the polymer chain length increases, more ester functional groups become available for cation interaction allowing the polymer to more efficiently coordinate the cation(s). As the PBA chain increases from DP $=1$ to DP $=5$, the peak width of the IM plot increases in size. A larger peak width could indicate either the presence multiple conformations or the existence of a conformational change during the IM separation.

\subsection{Statistical Analysis of Charge State $+1,+2$, and +3}

To better understand coordination similarities among PBA-cation DP groups, we performed single-factor analysis of variance (ANOVA) on CCS values obtained for each DP along the various charge states. ANOVA was used to compare PBA-Li, PBA-Na, PBA-K, PBA-Rb, and PBA-Cs species to one another. In Figure 4, the CCS values are compared against the same DP chain length, and as notated previously are observed to converge towards the same $\mathrm{CCS}$ as the DP chain length increases from $\mathrm{DP}=1$ to $\mathrm{DP}=5$. While converging of CCS values is clearly observed for charge state +1 in Figure 4, we investigated CCS trends within charge state +2 and +3 which are somewhat more difficult to interpret. In Figure 2, the DP chain lengths represent oligomers ranging from $\mathrm{DP}=1-43$ among the three charge states observed; each CCS value was measured in triplicate for all 5 metal ions, totaling 1,125 data points. In this situation, it is best to use statistical methodology to check for significant differences, and this present problem is well suited for single-factor ANOVA. ANOVA compares the variance between CCS values measured for each of the different metal ions variance (between) with the collective variance from the reproducibility in measurement of each metal cations CCS value (within). The F ratio of the variances, $\mathrm{F}=$ variance (between) / variance (within), is compared to the critical $\mathrm{F}$ value using the appropriate degrees of freedom in the two variables: 4 and 10, respectively. The results were compared to determine if they were significant at the $95 \%$ confidence level $(a=0.05)$; this confidence level is typically chosen for scientific experiments. The ANOVA results identified structural differences and similarities between the polymer topologies in the gas-phase that were not qualitatively evident. A detailed description and example of the ANOVA test conducted in this study can be found in Supporting Information on Pages S8-S9 and in Figure S5.

In Table 2 for charge state +1 species, the CCS values for each PBA-cation measured between DP $=1-3$ are determined to be significantly different. However, the CCS values 
for PBA-cations at DP $=4-11$ are considered to be significantly the same at the $95 \%$ confidence level. This is consistent with the data of Figure 4. Raw data and detailed statistical analysis for charge state +1 trend can be found in Supporting Information (Figure S6). For charge state +2 , the CCS values for PBA-cation species between DP $=4-6$ are significantly different, but significantly the same for $\mathrm{DP}=7-22$. Note, only charge state +2 PBA-Li, PBA-Na, and PBA-K species for DP $=4$ were detected. Larger cations $\mathrm{Rb}$ and $\mathrm{Cs}$ were observed to cationize with PBA starting at $\mathrm{DP}=5$ chain length.

In Figure 2, a folding region is observed for both the +2 and +3 charge states. When investigating the +3 charge state in detail, unique behavior was noted for the PBA-Li species during the structural transition region (described below). The +3 charge state trend is quite different from the other charge state trends. Here, all five of the PBA-cation species have significantly different CCS values for the metal cations between DP $=7-25$. However, inspection indicated that the CCS values for $\mathrm{Li}+3$ are typically smaller than the $+3 \mathrm{CCS}$ values for the other four metal cations. When the PBA-Li data are removed from the data set, the remaining PBA-cation ( $\mathrm{Na}, \mathrm{K}, \mathrm{Rb}$, and $\mathrm{Cs}$ ) $\mathrm{CCS}$ values are considered to be significantly different for $\mathrm{DP}=9-11$, to be the same for $\mathrm{DP}=12-13$, different for $\mathrm{DP}=$ $14-16$, and significantly the same for $\mathrm{DP}=17-24$, with one outlier at $\mathrm{DP}=25$. As the PBA chain length increases beyond DP $=25$, PBA-Cs is not detected in the IM-MS spectra. Interestingly, given the odd behavior of $\mathrm{Li}$ for the +3 charge state structural transition region, here we observed significant similarities among PBA-cation ( $\mathrm{Li}, \mathrm{Na}, \mathrm{K}$, and $\mathrm{Rb}$ ) species between $\mathrm{DP}=26-29$, and structural differences for $\mathrm{DP}=30$. The ANOVA test aided in differentiating the similarities and differences between PBA-cation CCS means and their gas-phase behavior. Raw data used for ANOVA test can be found in Supporting Information Figures S7-S12, for charge states +2 and +3 .

\subsection{Mass Targeted Experiments}

Previous MALDI-TOF/TOF CID studies explored cyclic and linear PBA low energy fragmentation mechanisms using $\mathrm{Li}$ and $\mathrm{Na}$ cations [39]. In this study, we explored the unique fragmentation behavior of each alkali metal cation $(\mathrm{Li}, \mathrm{Na}, \mathrm{K}, \mathrm{Rb}$, and $\mathrm{Cs}$ ) for $\mathrm{DP}=$ 1 and $\mathrm{DP}=5$ in the +1 charge state. When characterizing $\mathrm{DP}=1$ species, informative fragment ions were observed only for PBA-Li species as seen in Supporting Information Figure S13, with unique fragment ion structures attributed to PBA-Li species outlined in Supporting Information Figure S14. Here, at relatively low energy, PBA-Li is observed to produce fragment ions attributed to 1,5 $\mathrm{H}$-shift fragmentation. The small atomic radius of $\mathrm{Li}$, aids in its ability to fit within the polyester pocket, stabilizing the charge on the fragment ion. When monitoring PBA-Na, PBA-K, PBA-Rb, and PBA-Cs for DP $=1$, fragment ions were not observed. Instead each PBA-cationized species was observed to lose its respective cation as CID energy increased. IM-MS/MS fragmentation spectra for each PBA-cationized species for DP = 1 can be found in Supporting Information Figure S13, S15-S16.

When monitoring CID energy with respect to $\mathrm{DP}=5$, we observed unique fragment ions for both PBA-Li and PBA-Na species. Figure 5a illustrates 1,5 H-shift fragmentation, commonly referred to as a McLafferty Rearrangement mechanism, where fragment ions result from a single chain fracture. There are only two series of peaks that can be formed 
from the 1,5 H-shift of a symmetrical PBA precursor. Fragmentation occurs by hydrogen transfer from the diol chain to the ester carbonyl, but not from the acid segment. Both PBA$\mathrm{Li}$ and PBA-Na species were observed to undergo 1,5 $\mathrm{H}$-shift, promoting fragmentation at the $\mathrm{C}(\mathrm{O}) \mathrm{O}-\mathrm{CH} 2$ bond generating butanol-carboxyl and butanol-butene species. Both sets of fragment ions from the 1,5 H-shift were observed in the MS/MS spectra, as seen in Supporting Information Figure S17.

Figure $5 \mathrm{~b}$ illustrates the 1,3 $\mathrm{H}$-shift, which was also observed for both PBA-Li and PBA-Na species. This hydrogen transfer reaction causes fragmentation at the $\mathrm{C}(\mathrm{O})-\mathrm{O}$ bond producing two stable fragments: (1) diol terminus and (2) ketene end group. These results correspond to what has previously been reported regarding DP $=5$ PBA-Li and PBA-Na species. Fragment ion series (i.e. PBA-Li: 297, 497, 697, etc. and PBA-Na: 213, 413, 613, etc.) associated with -OH terminus in the 1,3 H-shift pathway, are in low abundance for ESI-MS. In previous MALDI-MS studies, this 1,3 H-shift fragment ion series was observed at higher signal intensity, this difference in peak intensity can most likely be attributed to ionization differences between the MALDI and ESI process.

Respective fragment ions $(\mathrm{m} / \mathrm{z})$ and their mechanistic pathways observed for both PBA-Li and PBA-Na species can be found in Supporting Information Figure S18. IM-MS/MS spectra of PBA-Li and PBA-Na are shown in Figure 5c-d. Fragment ion generation was monitored for each PBA-cationized species at CID energies $0-110 \mathrm{~V}$ in $10 \mathrm{~V}$ increments; see Supporting Information Figures S19-S20. In Figure 5c-d, CID spectra for 0 V, 30 V, and $60 \mathrm{~V}$ have dotted lines drawn around fragment ions and precursor species. As similarly seen with DP = 1 PBA-Li species, there were no fragment ions detected for PBA-K, PBA-Rb, and PBA-Cs, these species preferentially lost their respective cation as CID energy increased. We observe both PBA-Li and PBA-Na species to undergo charge remote 1,5 $\mathrm{H}$-shift and 1,3 $\mathrm{H}$-shift fragmentation as CID energy increases.

In this study, no unique fragment ions were observed for either Li or Na cationized PBA. However, Li did produce more intense fragment ion peaks relative to PBA-Na fragment ions. In addition to 1,5 $\mathrm{H}$-shift and 1,3 $\mathrm{H}$-shift fragment ions, other fragment ions are formed such as a di-acid (carboxyl end groups), most likely formed by an intra-chain fragmentation mechanism. Moreover, this data is consistent with the previously reported MALDITOF/TOF CID studies [39].

\section{Conclusions}

In this study, PBA $\left(M_{n}=2250\right)$ has been extensively characterized with monovalent alkali metal cations ( $\mathrm{Li}, \mathrm{Na}, \mathrm{K}, \mathrm{Rb}$, and $\mathrm{Cs}$ ) using IM-MS, IM-MS/MS, and statistical analysis. Unique charge state trend lines were observed for this polymer and it was concluded that these structural trends were native to the polymer and not specific to either the cationizing agents or instrumentation used in this study. Charge state $+1,+2$, and +3 trend lines increased in CCS respective to increasing PBA chain length. Regions of structural transitions representing gas-phase polymer folding were more distinctly observed for charge states +2 and +3 , although PBA-cations $\mathrm{Na}, \mathrm{K}, \mathrm{Rb}$, and $\mathrm{Cs}$ were also observed to undergo structural transitions along the +1 charge state trend line. The tipping point at which the gas- 
phase structural behavior begins to change was determined to be the same for each cation tested within the same charge state trend line. ANOVA statistical analysis aided in evaluating unique gas-phase behavior of the PBA-cations. Significant differences and similarities between PBA-cation CCS means, were compared to characterize gas-phase behavior. Tandem IM-MS/MS revealed that PBA-Li and PBA-Na species preferentially promote charge remote fragmentation to produce 1,5 $\mathrm{H}$-shift and 1,3 $\mathrm{H}$-shift fragment ions at $\mathrm{DP}=5$. Other PBA-cation species ( $\mathrm{K}, \mathrm{Rb}$, and $\mathrm{Cs}$ ) preferentially lost their cationizing agent as CID energy increased yielding no structurally useful information.

\section{Supplementary Material}

Refer to Web version on PubMed Central for supplementary material.

\section{Acknowledgments}

We thank Hartmut Nefzger and Bayer Material Sciences for providing the poly(butylene adipate) $\left(M_{n}=2250\right)$ sample. This work was funded and supported in part by the resources of the Center for Innovative Technology (CIT) at Vanderbilt University. Financial support for this research was provided by the National Institutes of Health (NIH NIGMS R01GM092218 and NIH NCI 1R03CA222452-01).

\section{References}

[1]. Oertel G, Polyurethane handbook, Carl Hanser Verlag, 1985.

[2]. Oschmann B, Lawrence J, Schulze MW, Ren JM, Anastasaki A, Luo Y, Nothling MD, Pester CW, Delaney KT, Connal LA, McGrath AJ, Clark PG, Bates CM, Hawker CJ, Effects of tailored dispersity on the self-assembly of dimethylsiloxane-methyl methacrylate block co-oligomers, ACS Macro Lett. 6 (2017) 668-673. doi:10.1021/acsmacrolett.7b00262.

[3]. Crotty S, Gerişlioğlu S, Endres KJ, Wesdemiotis C, Schubert US, Polymer architectures via mass spectrometry and hyphenated techniques: A review, Anal. Chim. Acta 932 (2016) 1-21. doi: 10.1016/j.aca.2016.05.024. [PubMed: 27286765]

[4]. Hoskins JN, Trimpin S, Grayson SM, Architectural differentiation of linear and cyclic polymeric isomers by ion mobility spectrometry-mass spectrometry, Macromolecules. 44 (2011) 69156918. doi:10.1021/ma2012046.

[5]. Montenegro-Burke JR, Bennett JM, McLean JA, Hercules DM, Novel behavior of the chromatographic separation of linear and cyclic polymers, Anal. Bioanal. Chem 408 (2016) $677-$ 681. doi:10.1097/NCN.0b013e3181a91b58.Exploring. [PubMed: 26637218]

[6]. Wesdemiotis C, Multidimensional mass spectrometry of synthetic polymers and advanced materials, Angew. Chemie Int. Ed 56 (2017) 1452-1464. doi:10.1002/anie.201607003.

[7]. Forsythe JG, Stow SM, Nefzger H, Kwiecien NW, May JC, McLean JA, Hercules DM, Structural characterization of methylenedianiline regioisomers by ion mobility-mass spectrometry, tandem mass spectrometry, and computational strategies: I. electrospray spectra of 2-ring isomers, Anal. Chem 86 (2014) 4362-4370. doi:10.1021/ac5001652. [PubMed: 24678803]

[8]. Gies AP, Hercules DM, Collision induced dissociation study of ester-based polyurethane fragmentation reactions, Anal. Chim. Acta 808 (2014) 199-219. doi:10.1016/j.aca.2013.09.035. [PubMed: 24370105]

[9]. Barrere C, Selmi W, Hubert-Roux M, Coupin T, Assumani B, Afonso C, Giusti P, Rapid analysis of polyester and polyethylene blends by ion mobility-mass spectrometry, Polym. Chem 5 (2014) 3576-3582. doi:10.1039/c4py00164h.

[10]. Alexander NE, Swanson JP, Joy A, Wesdemiotis C, Sequence analysis of cyclic polyester copolymers using ion mobility tandem mass spectrometry, Int. J. Mass Spectrom. 429 (2018) 151-157. doi:10.1016/j.ijms.2017.07.019. 
[11]. Liu X, Cool LR, Lin K, Kasko AM, Wesdemiotis C, Tandem mass spectrometry and ion mobility mass spectrometry for the analysis of molecular sequence and architecture of hyperbranched glycopolymers, Analyst. 140 (2015) 1182-1191. doi:10.1039/c4an01599a. [PubMed: 25519163]

[12]. Arcella A, Portella G, Ruiz ML, Eritja R, Vilaseca M, Gabelica V, Orozco M, Structure of triplex DNA in the gas phase, J. Am. Chem. Soc 134 (2012) 6596-6606. doi:10.1021/ja209786t. [PubMed: 22420643]

[13]. Koomen JM, Ruotolo BT, Gillig KJ, McLean JA, Russell DH, Kang M, Dunbar KR, Fuhrer K, Gonin M, Schultz JA, Oligonucleotide analysis with MALDI-ion-mobility-TOFMS, Anal. Bioanal. Chem 373 (2002) 612-617. doi:10.1007/s00216-002-1363-2. [PubMed: 12185573]

[14]. Burmistrova A, Gabelica V, Duwez AS, De Pauw E, Ion mobility spectrometry reveals duplex DNA dissociation intermediates, J. Am. Soc. Mass Spectrom. 24 (2013) 1777-1786. doi: 10.1007/s13361-013-0721-y. [PubMed: 24009017]

[15]. Fenn LS, Kliman M, Mahsut A, Zhao SR, McLean JA, Characterizing ion mobility-mass spectrometry conformation space for the analysis of complex biological samples, Anal. Bioanal. Chem 394 (2009) 235-244. doi:10.1007/s00216-009-2666-3. [PubMed: 19247641]

[16]. Fenn LS, McLean JA, Structural resolution of carbohydrate positional and structural isomers based on gas-phase ion mobility-mass spectrometry, Phys. Chem. Chem. Phys 13 (2011) 21962205. doi:10.1039/c0cp01414a. [PubMed: 21113554]

[17]. McLean JA, Ruotolo BT, Gillig KJ, Russell DH, Ion mobility-mass spectrometry: A new paradigm for proteomics, Int. J. Mass Spectrom. 240 (2005) 301-315. doi:10.1016/j.ijms. 2004.10.003.

[18]. Von Helden G, Bowers MT, Wyttenbach T, Inclusion of a MALDI ion source in the ion chromatography technique: conformational information on polymer and biomolecular ions, Int. J. Mass Spectrom. Ion Process. 147 (1995) 349-364.

[19]. Wyttenbach T, von Helden G, Bowers MT, Conformations of alkali ion cationized polyethers in the gas phase: polyethylene glycol and bis[(benzo-15-crown-5)-15-ylmethyl] pimelate, Int. J. Mass Spectrom. Ion Process. 165 (1997) 377-390. doi:10.1016/S0168-1176(97)00179-1.

[20]. F. de la M. J Criado-Hidalgo Ernesto, Fernández-García Juan, Mass and charge distribution analysis in negative electrosprays of large polyethylene glycol chains by ion mobility mass spectrometry, Anal. Chem 85 (2013) 2710-2716. doi:10.1021/ac303054x. [PubMed: 23317444]

[21]. Gidden J, Jackson AT, Scrivens JH, Bowers MT, Gas phase conformations of synthetic polymers: poly(methyl methacrylate) oligomers cationized by sodium ions, Int. J. Mass Spectrom. 188 (1999) 121-130. doi:10.1016/S1387-3806(98)14287-2.

[22]. Gidden J, Bowers MT, Jackson AT, Scrivens JH, Gas-phase conformations of cationized poly(styrene) oligomers, J. Am. Soc. Mass Spectrom. 13 (2002) 499-505. doi:10.1016/ S1044-0305(02)00367-7. [PubMed: 12019974]

[23]. Gidden J, Wyttenbach T, Batka JJ, Weis P, Bowers MT, Jackson AT, Scrivens JH, Poly(ethylene terephthalate) oligomers cationized by alkali ions: Structures, energetics, and their effect on mass spectra and the matrix-assisted laser desorption/ionization process, J. Am. Soc. Mass Spectrom. 10 (1999) 883-895. doi:10.1016/S1044-0305(99)00054-9.

[24]. Morsa D, Defize T, Dehareng D, Jérôme C, De Pauw E, Polymer topology revealed by ion mobility coupled with mass spectrometry, Anal. Chem 86 (2014) 9693-9700. doi:10.1021/ ac502246g. [PubMed: 25188877]

[25]. Haler JRN, Morsa D, Lecomte P, Jérôme C, Far J, De Pauw E, Predicting ion mobility-mass spectrometry trends of polymers using the concept of apparent densities, Elsevier Methods. 144 (2018) 125-133. doi:10.1016/j.ymeth.2018.03.010.

[26]. Foley CD, Zhang B, Alb AM, Trimpin S, Grayson SM, Use of ion mobility spectrometry-mass spectrometry to elucidate architectural dispersity within star polymers, ACS Macro Lett 4 (2015) 778-782. doi:10.1021/acsmacrolett.5b00299.

[27]. Kim K, Lee JW, Chang T, Kim HI, Characterization of polylactides with different stereoregularity using electrospray ionization ion mobility mass spectrometry, J. Am. Soc. Mass Spectrom. 25 (2014) 1771-1779. doi:10.1007/s13361-014-0949-1. [PubMed: 25001385]

[28]. Duez Q, Josse T, Lemaur V, Chirot F, Choi CM, Dubois P, Dugourd P, Cornil J, Gerbaux P, De Winter $\mathrm{J}$, Correlation between the shape of the ion mobility signals and the stepwise folding 
process of polylactide ions, J. Mass Spectrom. 52 (2017) 133-138. doi:10.1002/jms.3915. [PubMed: 28112477]

[29]. Trimpin S, Plasencia M, Isailovic D, Clemmer DE, Resolving oligomers from fully grown polymers with IMS-MS, Anal. Chem 79 (2007) 7965-7974. doi:10.1021/ac071575i. [PubMed: 17887728]

[30]. Trimpin S, Clemmer DE, Ion Mobility Spectrometry / Mass Spectrometry Snapshots for Assessing the Molecular Compositions of Complex Polymeric Systems, Anal. Chem 80 (2008) 9073-9083. [PubMed: 19551934]

[31]. Ude S, Fernández De La Mora J, Thomson BA, Charge-induced unfolding of multiply charged polyethylene glycol ions, J. Am. Chem. Soc 126 (2004) 12184-12190. doi:10.1021/ja0381306. [PubMed: 15382955]

[32]. Nefzger H, Personal communication, 2011.

[33]. Chakraborty A, Mass spectrometric study of poly(1,4-butylene adipate)-based model polyurethanes, Vanderbilt University, 2012.

[34]. May JC, Goodwin CR, Lareau NM, Leaptrot KL, Morris CB, Kurulugama RT, Mordehai A, Klein C, Barry W, Darland E, Overney G, Imatani K, Stafford GC, Fjeldsted JC, McLean JA, Conformational ordering of biomolecules in the gas phase: Nitrogen collision cross sections measured on a prototype high resolution drift tube ion mobility-mass spectrometer, Anal. Chem 86 (2014) 2107-2116. doi:10.1021/ac4038448. [PubMed: 24446877]

[35]. May JC, Dodds JN, Kurulugama RT, Stafford GC, Fjeldsted JC, McLean JA, Broadscale resolving power performance of a high precision uniform field ion mobility-mass spectrometer, Analyst. 140 (2015) 6824-6833. doi:10.1039/c5an00923e. [PubMed: 26191544]

[36]. Stow SM, Causon TJ, Zheng X, Kurulugama RT, Mairinger T, May JC, Rennie EE, Baker ES, Smith RD, McLean JA, Hann S, Fjeldsted JC, An interlaboratory evaluation of drift tube ion mobility - mass spectrometry collision cross section measurements, 89 (2017) 9048-9055. doi: 10.1186/s40945-017-0033-9.Using.

[37]. Gabelica V, Shvartsburg AA, Afonso C, Barran P, Benesch JLP, Bleiholder C, Bowers MT, Bilbao A, Bush MF, Campbell JL, Campuzano IDG, Causon T, Clowers BH, Creaser CS, De Pauw E, Far J, Fernandez-Lima F, Fjeldsted JC, Giles K, Groessl M, Jr CJH, Hann S, Kim HI, Kurulugama RT, May JC, McLean JA, Pagel K, Richardson K, Ridgeway ME, Rosu F, Sobott F, Thalassinos K, Valentine SJ, Wyttenbach T, Recommendations for reporting ion mobility Mass Spectrometry measurements, Mass Spectrom. Rev (2019) 1-30. doi:10.1002/mas.21585.

[38]. Gabelica V, Livet S, Rosu F, Optimizing native ion mobility Q-TOF in helium and nitrogen for very fragile noncovalent structures, J. Am. Soc. Mass Spectrom. 29 (2018) 2189-2198. doi: 10.1007/s13361-018-2029-4. [PubMed: 30047072]

[39]. Gies AP, Ellison ST, Chakraborty AK, Kwiecien NW, Hercules DM, MALDI-TOF/TOF CID study of poly(butylene adipate) fragmentation reactions, RSC Adv. 2 (2012) 4135-4151. doi: $10.1039 / \mathrm{c} 2 \mathrm{ra} 20283 \mathrm{~b}$. 


\section{Highlights}

- Combination of monovalent alkali cations ( $\mathrm{Li}, \mathrm{Na}, \mathrm{K}, \mathrm{Rb}$, and $\mathrm{Cs}$ ) were used to structurally characterize PBA oligomers.

- This material was characterized via IM-MS, IM-MS/MS, and ANOVA.

- Unique charge state trends $(\mathrm{z}=+1,+2$, and +3$)$ were extracted for each cation-adducted PBA oligomer.

- $\quad$ Unique fragment ions were only observed for PBA cationized to Li and Na; the PBA precursor preferentially lost the larger cations (K, Rb, and $\mathrm{Cs})$ as activation energy increased. 
<smiles>CC(C)OCCCCOC(=O)CCCCC(=O)OCCCCO</smiles>

Figure 1.

Structure of telechelic polybutylene adipate (PBA). 


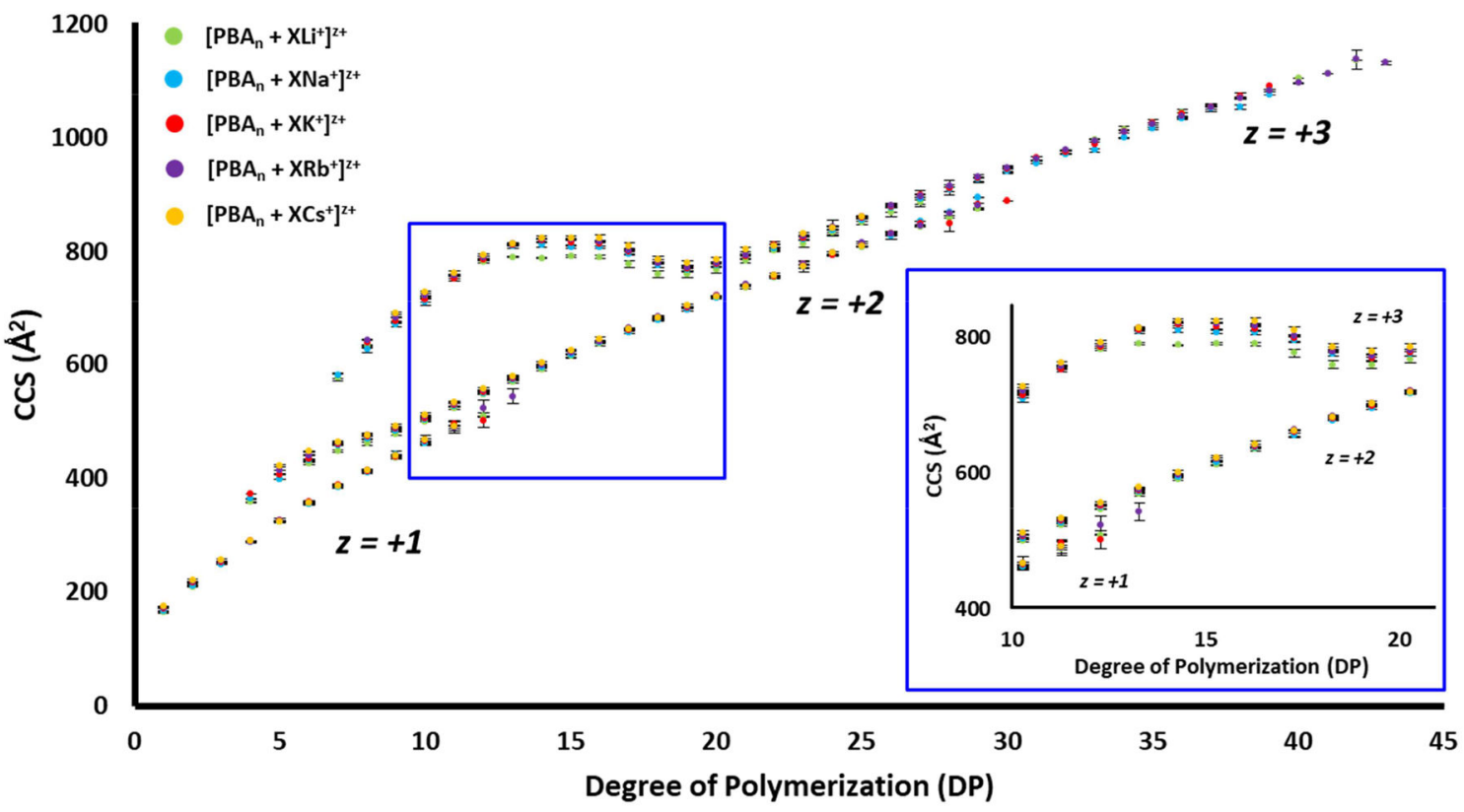

Figure 2.

IM-MS mobility plot of CCS versus degree of polymerization (DP) of cationized linear PBA oligomers ( $\mathrm{n}$ ) for different charge states $(\mathrm{z}=+1,+2$, and +3$)$. Cations used to ionize PBA are annotated by color $(\mathrm{Li}=$ green, $\mathrm{Na}=$ blue, $\mathrm{K}=$ red, $\mathrm{Rb}=$ purple, and $\mathrm{Cs}=$ yellow $)$, where $\mathrm{X}$ is the number of cations ionizing PBAn. 

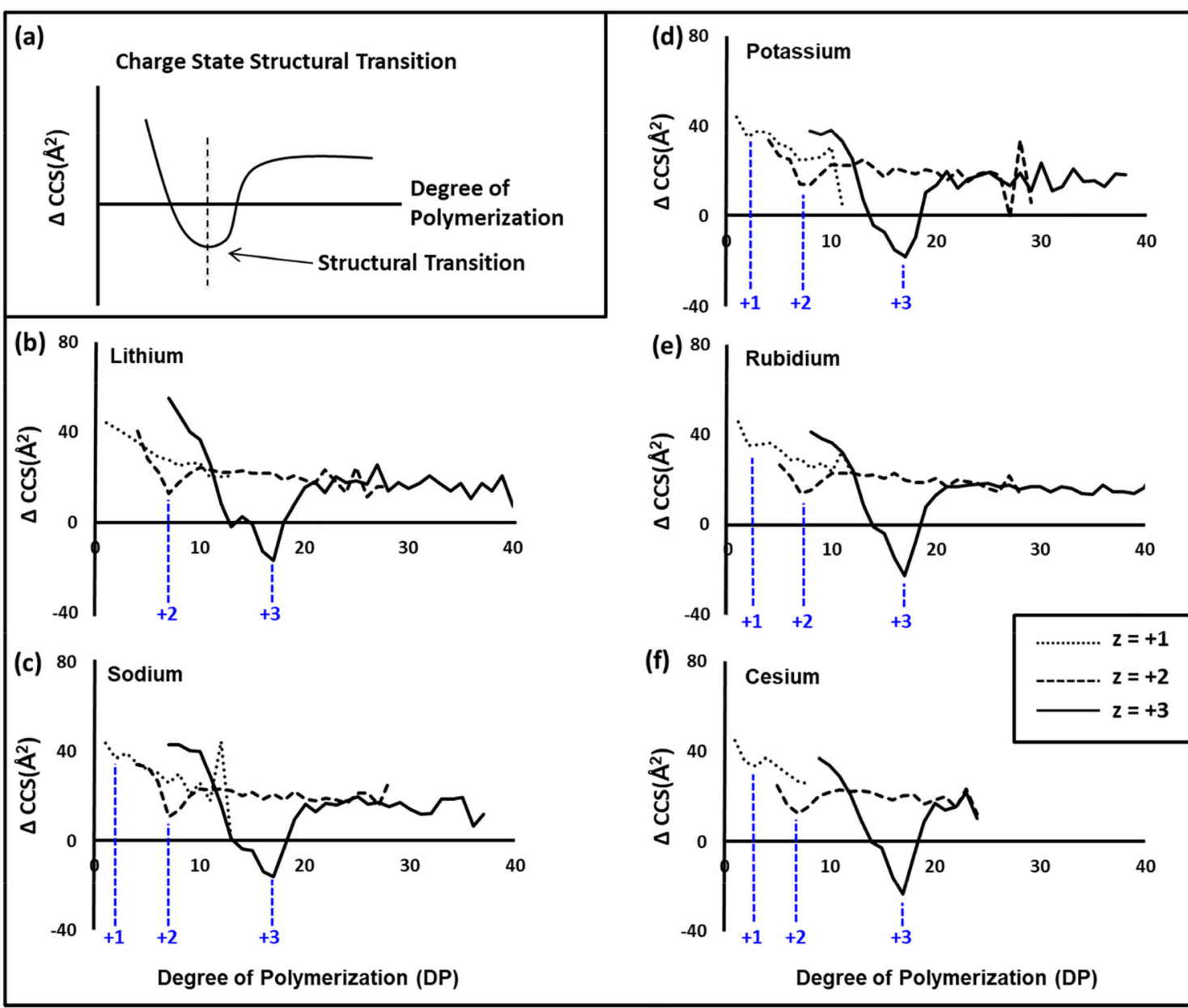

Figure 3.

Plots of $\triangle \mathrm{CCS}$ vs DP which assist in monitoring gas-phase structural trends. (a) Conceptual illustration of a polymer undergoing charge dependent structural transition as indicated by a well-defined negative apex. (b) Charge state trends observed for each cationized PBA DP include: $\mathrm{z}=+1$ (dotted line), $\mathrm{z}=+2$ (dashed line), and $\mathrm{z}=+3$ (solid line). Blue dashed lines are drawn for visual alignment of transition. 
(a) $\mathrm{DP}=1$

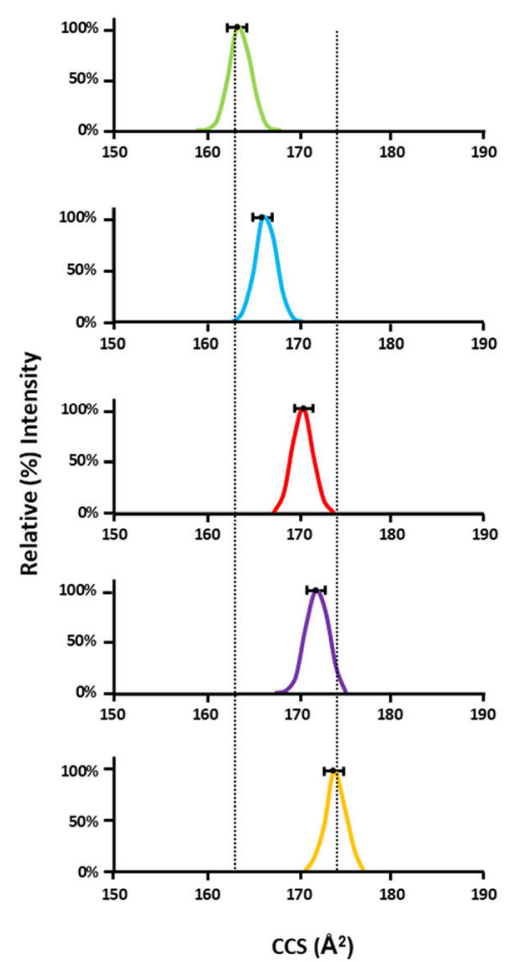

(b) $\mathrm{DP}=3$
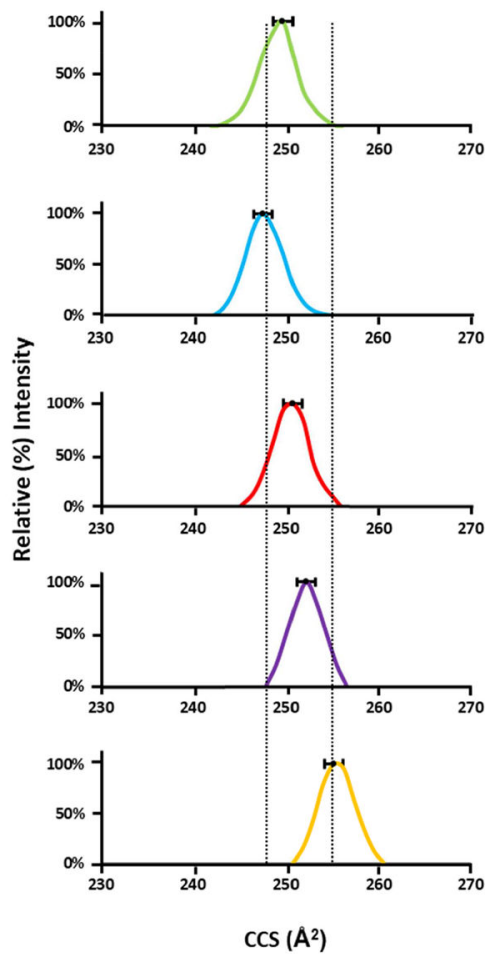

(c) $\mathrm{DP}=5$

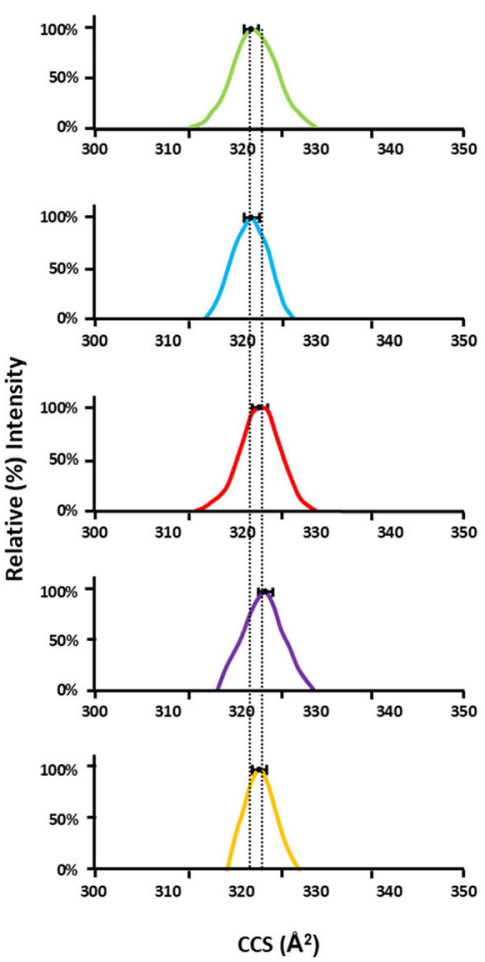

Figure 4.

IM plots of PBA-cations having charge state +1 are shown above. Experimental CCS and their respective standard deviations are given for (a) $\mathrm{DP}=1$, (b) $\mathrm{DP}=3$, and (c) $\mathrm{DP}=5$. Cations are notated by color: $\mathrm{Li}=$ green, $\mathrm{Na}=$ blue, $\mathrm{K}=$ red, $\mathrm{Rb}=$ purple, and $\mathrm{Cs}=$ yellow . Dotted lines are drawn for visual alignment representing maximum and minimum CCS values. 
(a)
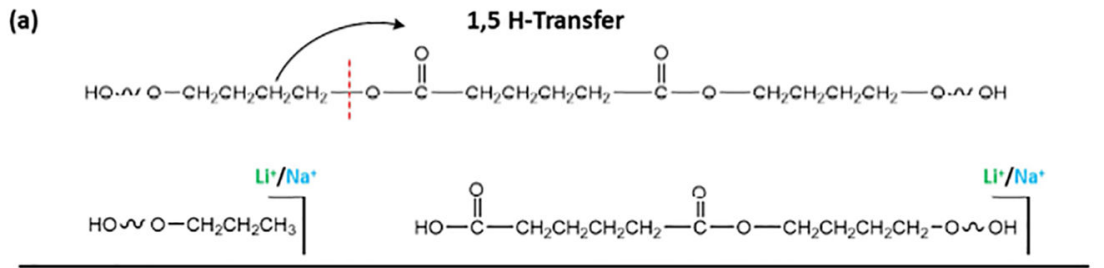

(b)

1,3 H-Transfer
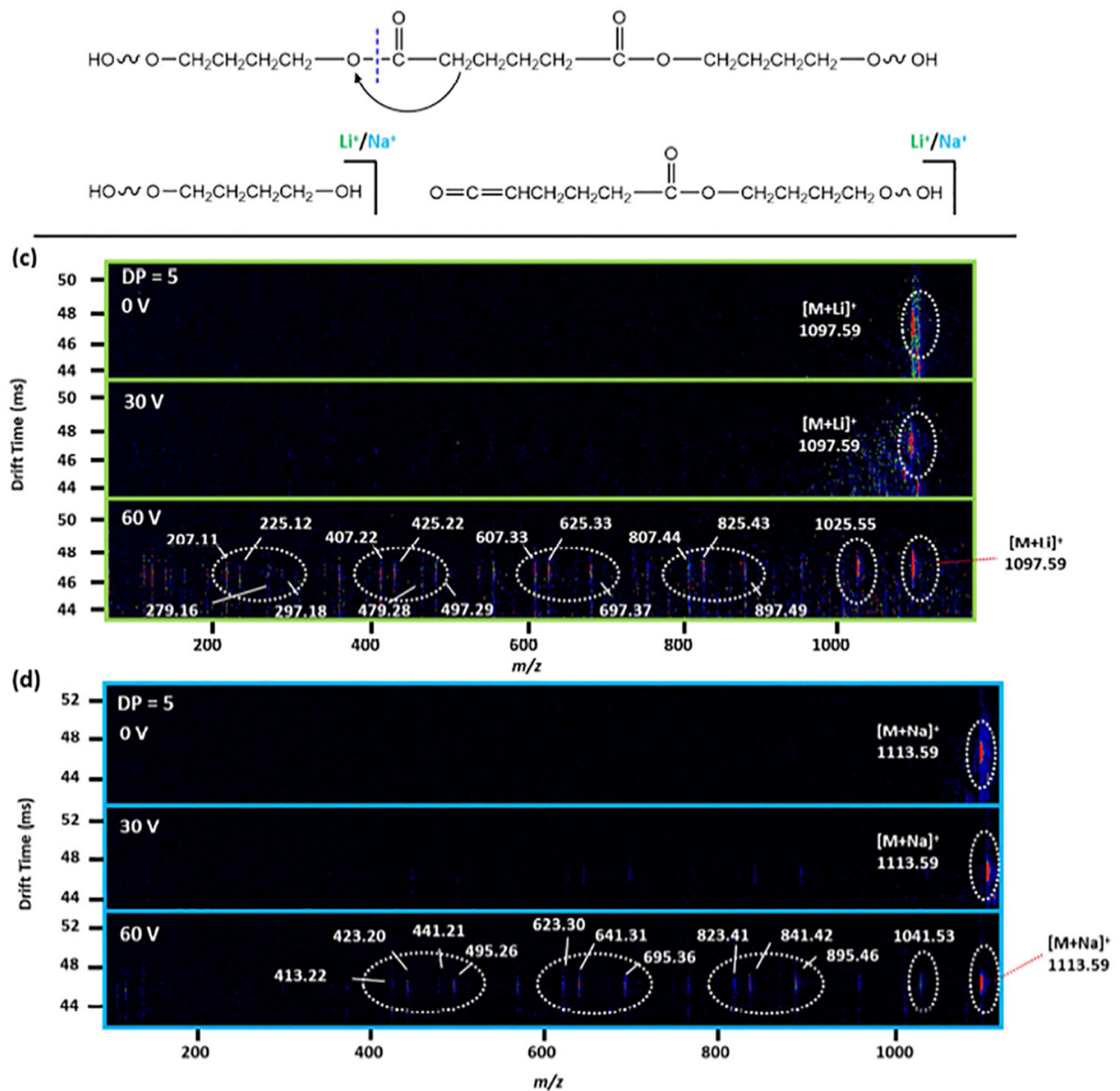

Figure 5.

Fragmentation reaction mechanisms and results for singly charged PBA DP $=5$. (a) 1,5 Hshift and (b) 1,3 H-shift produces two stable fragment ions. (c) IM-MS/MS spectrum for PBA-Li at 0, 30, and $60 \mathrm{~V}$ collision energies and (d) IM-MS/MS spectrum for PBA-Na at 0, 30 , and $60 \mathrm{~V}$ collision energies. Respective fragment and precursor species are outlined. 


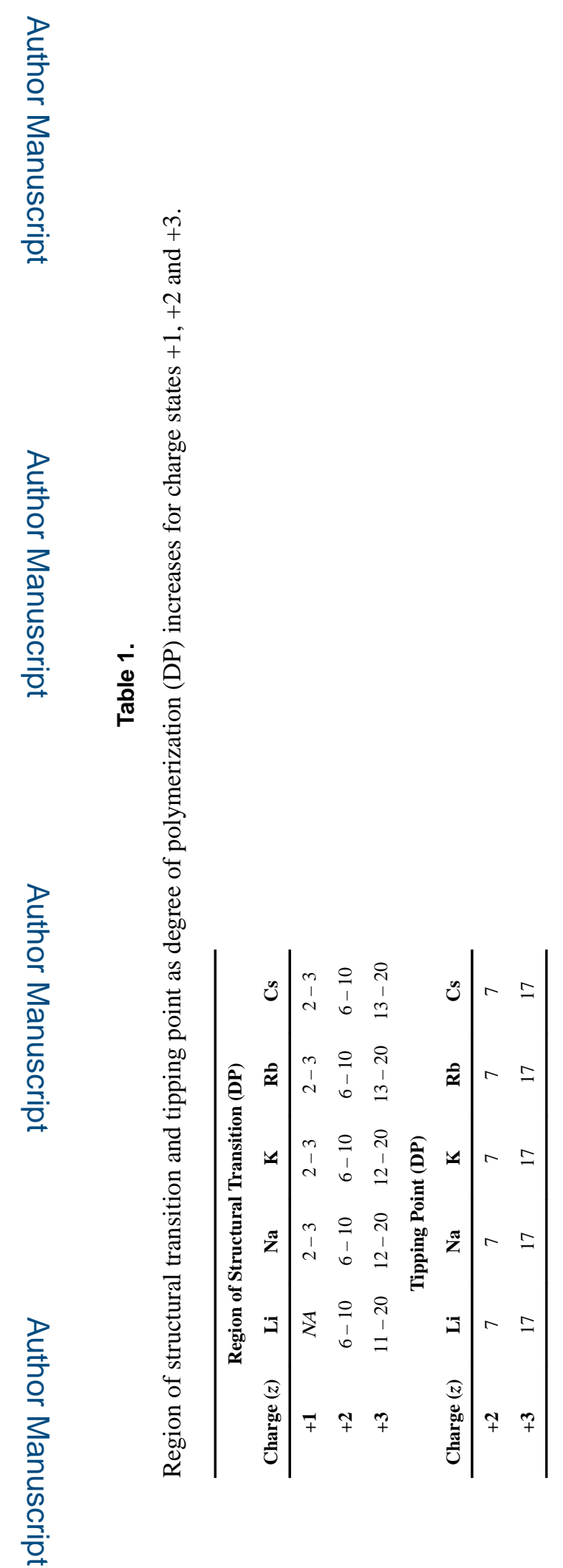

Polymer (Guildf). Author manuscript; available in PMC 2020 May 31. 


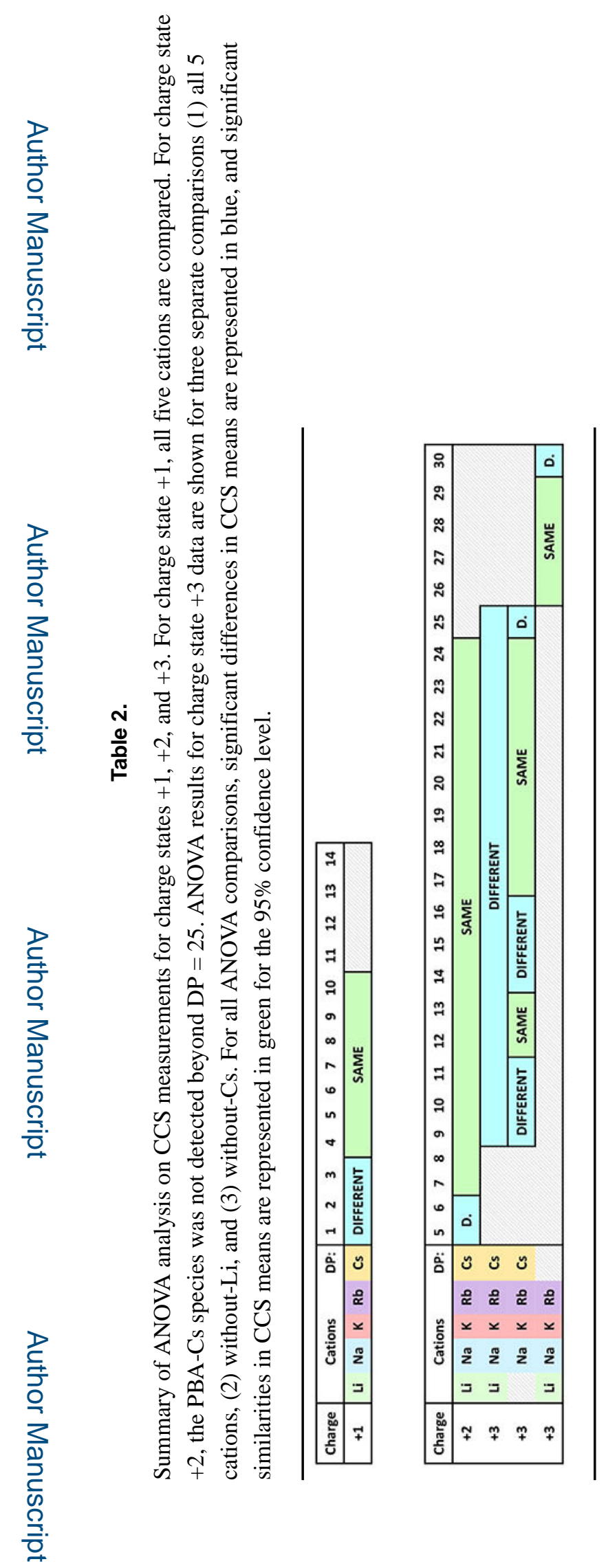

Polymer (Guildf). Author manuscript; available in PMC 2020 May 31. 